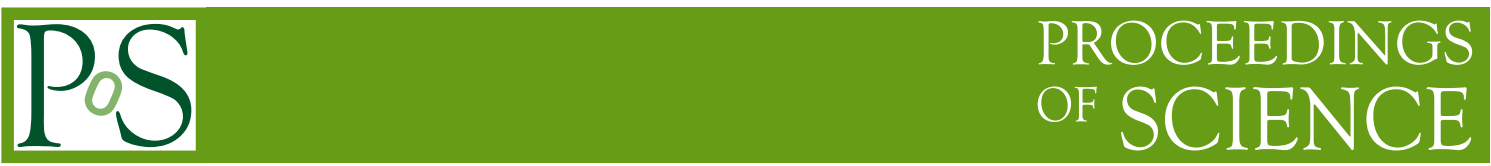

\title{
Nonperturbative results on the quark-gluon vertex
}

\author{
A. C. Aguilar* \\ Universidade Estadual de Campinas - UNICAMP \\ Instituto de Física Gleb Wataghin, 13083-859 - Campinas, SP, Brazil \\ E-mail: aguilareifi.unicamp.br

\section{Binosi} \\ European Centre for Theoretical Studies in Nuclear Physics and Related Areas (ECT*) \\ and Fondazione Bruno Kessler, \\ Villa Tambosi, Strada delle Tabarelle 286, I-38123 Villazzano (TN) Italy \\ E-mail: binosieectstar.eu
}

\section{J. C. Cardona}

Universidade Estadual de Campinas - UNICAMP

Instituto de Física Gleb Wataghin, 13083-859 - Campinas, SP, Brazil

E-mail: jeinerc@ifi.unicamp.br

\section{J. Papavassiliou}

Department of Theoretical Physics and IFIC, University of Valencia and CSIC,

E-46100, Valencia, Spain

E-mail: Joannis.Papavassiliou@uv.es

We present analytical and numerical results for the Dirac form factor of the quark-gluon vertex in the quark symmetric limit, where the incoming and outgoing quark momenta have the same magnitude but opposite sign. To accomplish this, we compute the relevant components of the quark-ghost scattering kernel at the one-loop dressed approximation, using as basic ingredients the full quark propagator, obtained as a solution of the quark gap equation, and the gluon propagator and ghost dressing function, obtained from large-volume lattice simulations.

Xth Quark Confinement and the Hadron Spectrum,

October 8-12, 2012

TUM Campus Garching, Munich, Germany

\footnotetext{
${ }^{*}$ Speaker.
} 


\section{Introduction}

In the last few years, considerable progress has been made in our understanding of the infrared (IR) behavior of the fundamental Green's functions of QCD, such as gluon, ghost, and quark propagators $[1,2,3,4,5,6,7,8,9]$, as well as some of the basic vertices of the theory $[6,10,11,12]$, and their relation to the confinement and dynamical chiral symmetry breaking (CSB) [8]. In fact, there is a broad consensus that one of the most important ingredients for the CSB is the non-abelian quark-gluon vertex, which controls the way the ghost sector enters into the gap equation. Specifically, this vertex introduces a numerically crucial dependence on the ghost dressing function and the quark-ghost scattering amplitude [13]. This latter quantity satisfies its own dynamical equation, which may be decomposed into individual integral equations for its various form factors. Here we will present the first steps towards the determination of the longitudinal quark-gluon vertex form factors for a particular kinematic configuration: the quark symmetric limit where the incoming and outgoing quark momenta have the same magnitude and opposite signs. To do that, we compute numerically the relevant quark-ghost scattering kernel components at the "one-loop dressed" approximation, at the same kinematic point, using as ingredients the nonperturbative lattice results for the gluon propagator and ghost dressing function of Ref. [2], and the solution of the quark gap equation obtained in [13] for the full quark propagator.

\section{Ingredients and definitions}

Consider the conventional quark gluon vertex shown in Fig. 1, and defined according to

$$
i \Gamma_{A_{\mu}^{a} \psi_{j} \bar{\psi}_{i}}\left(q, p_{2},-p_{1}\right)=i g t_{i j}^{a} \Gamma_{\mu}\left(q, p_{2},-p_{1}\right) ; \quad \Gamma_{\mu}^{(0)}\left(q, p_{2},-p_{1}\right)=\gamma_{\mu} ; \quad q+p_{2}=p_{1} .
$$

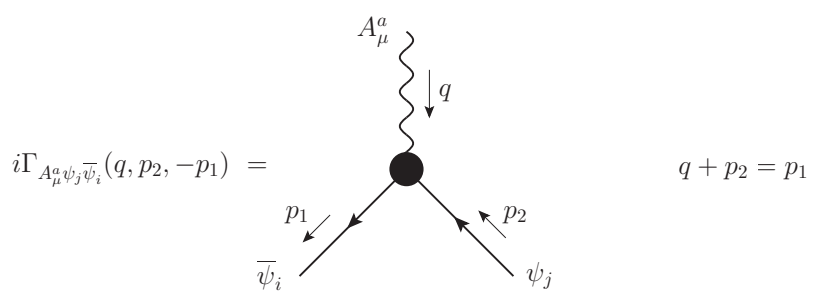

Figure 1: The conventional quark-gluon vertex with the momenta routing used throughout the text.

In the Batalin-Vilkoviski (BV) formalism, the Slavnov-Taylor identity (STI) satisfied by this vertex reads (in the kinematic configuration chosen) [5]

$$
q^{\mu} \Gamma_{A_{\mu}^{a} \psi_{j} \bar{\psi}_{i}}\left(q, p_{2},-p_{1}\right)=F\left(q^{2}\right)\left[\Gamma_{\psi_{k} \bar{\psi}_{i}}\left(p_{1}\right) \Gamma_{\psi_{j} c^{a} \bar{\psi}_{k}^{*}}\left(p_{2}, q,-p_{1}\right)+\Gamma_{\psi_{k}^{*} \bar{\psi}_{i} c^{a}}\left(p_{2},-p_{1}, q\right) \Gamma_{\psi_{j} \bar{\psi}_{k}}\left(p_{2}\right)\right] .
$$

In the formula above $F\left(q^{2}\right)$ denotes the so-called ghost dressing function which is related to the full ghost propagator $D^{a b}\left(q^{2}\right)$ through

$$
i D^{a b}\left(q^{2}\right)=i \delta^{a b} \frac{F\left(q^{2}\right)}{q^{2}} ; \quad F^{(0)}\left(q^{2}\right)=1,
$$


while $\Gamma_{\psi \bar{\psi}}$ is the inverse of the full fermion propagator $S_{i j}(p)$ obtained by solving the equation

$$
i S_{i j}(p) \Gamma_{\psi_{j} \bar{\psi}_{k}}=\delta_{i k} ; \quad i S_{i j}^{(0)}(p)=i \delta_{i j} S^{(0)}(p)=i \delta_{i j} \frac{1}{\not p-m} .
$$

The standard decomposition for the inverse of the full quark propagator $S^{-1}(p)$ is given by

$$
i S^{-1}(p)=i\left[A\left(p^{2}\right) \not p-B\left(p^{2}\right)\right],
$$

where $A\left(p^{2}\right)$ and $B\left(p^{2}\right)$ are, respectively, the Dirac vector and scalar components.

In addition, $\psi_{k}^{*}$ and $\bar{\psi}_{k}^{*}$ represent the so-called antifields associated to the spinor fields $\bar{\psi}_{k}$ and $\psi_{k}$ respectively; they have ghost charge -1 , (mass) dimension 5/2, and obey Bose statistics. The Green's functions $\Gamma_{\psi_{k}^{*} \bar{\psi}_{i} c^{a}}$ and $\Gamma_{\psi_{j} c^{a} \bar{\psi}_{k}^{*}}$ are shown in Fig. 2.

These two functions are not independent, being related by "conjugation"; indeed, to get one from the other, we need to perform the following operations: (i) exchange $-p_{1}$ with $p_{2}:-p_{1} \leftrightarrow p_{2}$; (ii) reverse the sign of all external momenta: $q,-p_{1}, p_{2} \leftrightarrow-q, p_{1},-p_{2}$; (iii) take the hermitian conjugate of the resulting amplitude.

Then, introducing the function

$$
\begin{aligned}
H_{i j}^{a}\left(q, p_{2},-p_{1}\right) & =g t_{i j}^{a} H\left(q, p_{2},-p_{1}\right)=-i \Gamma_{\psi_{j} c^{c} \bar{\psi}_{k}^{*}}\left(p_{2}, q,-p_{1}\right), \\
\bar{H}_{i j}^{a}\left(-q, p_{1},-p_{2}\right) & =g t_{i j}^{a} \bar{H}\left(-q, p_{1},-p_{2}\right)=i \Gamma_{\psi_{k}^{*} \bar{\psi}_{i} c^{a}}\left(p_{2},-p_{1}, q\right),
\end{aligned}
$$

and factoring out a the common color and gauge coupling combination $g t_{i j}^{a}$, the STI (2.2) can be rewritten as

$$
q^{\mu} \Gamma_{\mu}\left(q, p_{2},-p_{1}\right)=F\left(q^{2}\right)\left[S^{-1}\left(p_{1}\right) H\left(q, p_{2},-p_{1}\right)-\bar{H}\left(-q, p_{1},-p_{2}\right) S^{-1}\left(p_{2}\right)\right],
$$

with $\bar{H}$ obtained from $H$ through the set of operations detailed above. The $H$ function admits the general form factor decomposition [14]

$$
H\left(q, p_{2},-p_{1}\right)=X_{0} \mathbb{I}+X_{1} \not p_{1}+X_{2} \not p_{2}+X_{3} \widetilde{\sigma}_{\mu v} p_{1}^{\mu} p_{2}^{v},
$$

where the form factors $X_{i}$ are functions of the momenta, $X_{i}=X_{i}\left(q^{2}, p_{2}^{2}, p_{1}^{2}\right)$ and $\widetilde{\sigma}_{\mu v}=1 / 2\left[\gamma_{\mu}, \gamma_{v}\right]$ (notice the $i$ difference with respect to the conventional definition of this quantity). One then obtains automatically the expansion

$$
\bar{H}\left(-q, p_{1},-p_{2}\right)=\bar{X}_{0} \mathbb{I}+\bar{X}_{2} \not p_{1}+\bar{X}_{1} \not p_{2}+\bar{X}_{3} \widetilde{\sigma}_{\mu \nu} p_{1}^{\mu} p_{2}^{v},
$$

where $\overline{X_{i}}=X_{i}\left(q^{2}, p_{1}^{2}, p_{2}^{2}\right)$.

At tree-level, one clearly has $X_{0}^{(0)}=\bar{X}_{0}^{(0)}=1$, with the remaining form factors vanishing.

The most general Lorentz decomposition for the longitudinal part of the vertex $\Gamma_{\mu}\left(q, p_{2},-p_{1}\right)$ appearing in Eq. (2.7) can be written as [14]

$$
\Gamma_{\mu}\left(q, p_{2},-p_{1}\right)=L_{1} \gamma_{\mu}+L_{2}\left(\not p_{1}+\not p_{2}\right)\left(p_{1}+p_{2}\right)_{\mu}+L_{3}\left(p_{1}+p_{2}\right)_{\mu}+L_{4} \widetilde{\sigma}_{\mu v}\left(p_{1}+p_{2}\right)^{v},
$$

where $L_{i}$ are the form factors, whose dependence on the momenta has been suppressed, in order to keep a compact notation, i.e., $L_{i}=L_{i}\left(q^{2}, p_{1}^{2}, p_{2}^{2}\right)$. Notice that the tree level expression for $\Gamma_{\mu}^{(0)}$ is recovered setting $L_{1}=1$ and $L_{2}=L_{3}=L_{4}=0$; then, $\Gamma_{\mu}^{(0)}=\gamma_{\mu}$. 


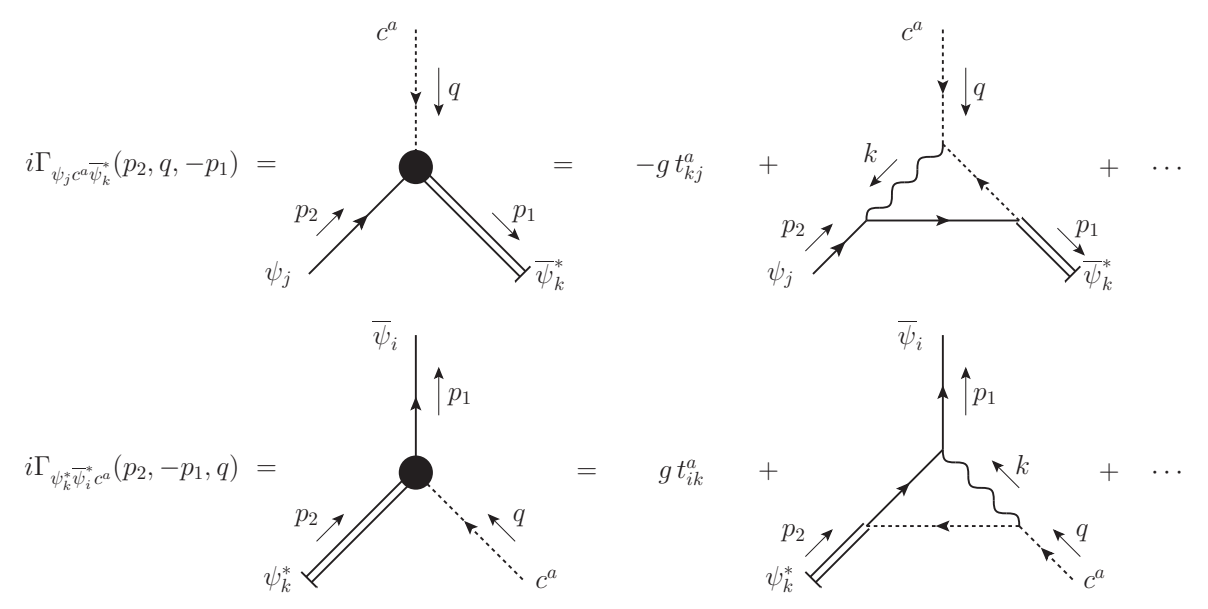

Figure 2: The auxiliary functions through which the STI satisfied by the quark-gluon vertex is satisfied. For convenience we show the momenta routing matching the kinematics chosen for the quark-gluon vertex as well as the tree-level and one-loop terms in the corresponding loop expansion.

Contracting Eq. (2.10) with $q^{\mu}$, we have

$$
q^{\mu} \Gamma_{\mu}=\left(p_{1}^{2}-p_{2}^{2}\right) L_{3} \mathbb{I}+\left[\left(p_{1}^{2}-p_{2}^{2}\right) L_{2}+L_{1}\right] p_{1}+\left[\left(p_{1}^{2}-p_{2}^{2}\right) L_{2}-L_{1}\right] p_{2}+2 L_{4} \widetilde{\sigma}_{\mu \nu} p_{1}^{\mu} p_{2}^{v} .
$$

In addition, substituting into Eq. (2.7) the full quark propagator $S^{-1}(p)$ of Eq. (2.5), and the expressions for $H$ and $\bar{H}$ given by Eqs. (2.8) and (2.9) respectively, we find that the rhs of Eq. (2.11) can be also expressed in terms of the functions $A, B$ and $X_{i}$ 's. Then, it is relatively straightforward to demonstrate that the $L_{i}$ 's may be expressed as [13]

$$
\begin{aligned}
L_{1} & =\frac{F(q)}{2}\left\{A\left(p_{1}\right)\left[X_{0}-\left(p_{1}^{2}+p_{1} \cdot p_{2}\right) X_{3}\right]+A\left(p_{2}\right)\left[\bar{X}_{0}-\left(p_{2}^{2}+p_{1} \cdot p_{2}\right) \bar{X}_{3}\right]\right\} \\
& +\frac{F(q)}{2}\left\{B\left(p_{1}\right)\left(X_{2}-X_{1}\right)+B\left(p_{2}\right)\left(\bar{X}_{2}-\bar{X}_{1}\right)\right\} ; \\
L_{2} & =\frac{F(q)}{2\left(p_{1}^{2}-p_{2}^{2}\right)}\left\{A\left(p_{1}\right)\left[X_{0}+\left(p_{1}^{2}-p_{1} \cdot p_{2}\right) X_{3}\right]-A\left(p_{2}\right)\left[\bar{X}_{0}+\left(p_{2}^{2}-p_{1} \cdot p_{2}\right) \bar{X}_{3}\right]\right\} \\
& -\frac{F(q)}{2\left(p_{1}^{2}-p_{2}^{2}\right)}\left\{B\left(p_{1}\right)\left(X_{1}+X_{2}\right)-B\left(p_{2}\right)\left(\bar{X}_{1}+\bar{X}_{2}\right)\right\} ; \\
L_{3} & =\frac{F(q)}{p_{1}^{2}-p_{2}^{2}}\left\{A\left(p_{1}\right)\left(p_{1}^{2} X_{1}+p_{1} \cdot p_{2} X_{2}\right)-A\left(p_{2}\right)\left(p_{2}^{2} \bar{X}_{1}+p_{1} \cdot p_{2} \bar{X}_{2}\right)-B\left(p_{1}\right) X_{0}+B\left(p_{2}\right) \bar{X}_{0}\right\} ; \\
L_{4} & =\frac{F(q)}{2}\left\{A\left(p_{1}\right) X_{2}-A\left(p_{2}\right) \bar{X}_{2}-B\left(p_{1}\right) X_{3}+B\left(p_{2}\right) \bar{X}_{3}\right\} .
\end{aligned}
$$

It is interesting to notice that setting in Eq. (2.12) $X_{0}=\bar{X}_{0}=1$ and $X_{i}=\bar{X}_{i}=0$, for $i \geq 1$, and $F(q)=1$, we obtain the following expressions

$$
L_{1}=\frac{A\left(p_{1}\right)+A\left(p_{2}\right)}{2}, \quad L_{2}=\frac{A\left(p_{1}\right)-A\left(p_{2}\right)}{2\left(p_{1}^{2}-p_{2}^{2}\right)}, \quad L_{3}=-\frac{B\left(p_{1}\right)-B\left(p_{2}\right)}{p_{1}^{2}-p_{2}^{2}}, \quad L_{4}=0 .
$$

which give rise to the so-called Ball-Chiu (BC) vertex [15], widely employed in the literature for studies of CSB [8]. 


\section{The "one-loop dressed" approximation for $\mathbf{H}$}

It is clear from Eq. (2.12), that in order to determine the longitudinal form factors $L_{i}$, it is necessary to know the nonperturbative behavior of the form factors $X_{i}$.

To obtain a nonperturbative estimate for $H$ and its form factors, we will study the "one-loop dressed" contribution represented in the diagram of Fig. 3, and given by

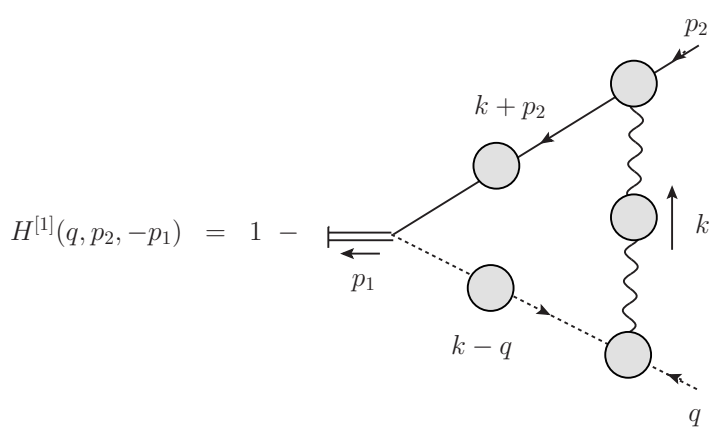

Figure 3: The quark-ghost scattering kernel at "one-loop dressed" approximation.

$$
H^{[1]}\left(q, p_{2},-p_{1}\right)=1-\frac{1}{2} i C_{A} g^{2} \int_{k} \Delta^{\mu v}(k) G_{v}(k-q) D(k-q) S\left(k+p_{2}\right) \Gamma_{\mu}\left(k, p_{2},-k-p_{2}\right),
$$

where $C_{A}$ is the eigenvalue of the Casimir operator in the adjoint representation, $\Delta^{\mu v}(q)$ is the full gluon propagator; in the Landau gauge

$$
i \Delta_{\mu \nu}^{a b}(q)=-i \delta^{a b}\left[g^{\mu v}-\frac{q^{\mu} q^{v}}{q^{2}}\right] \Delta(q) .
$$

For evaluating Eq. (3.1) further, we will use the following approximations: (i) the full gluonghost vertex will be replaced by its tree-level value, i.e. $i G_{v}^{a b c}=-g f^{a b c}(k-q)_{v}$. Note that, since the full $\Delta^{\mu v}(k)$ is transverse, only the $q_{v}$ part of the gluon-ghost survives since $k_{v} \Delta^{\mu v}(k)=0$; (ii) for the vertex $\Gamma_{\mu}$ we will use the following Ansatz

$$
\begin{aligned}
\Gamma_{\mu}\left(k, p_{2},-k-p_{2}\right) & =\frac{F(k)}{2}\left[A\left(p_{2}+k\right)+A\left(p_{2}\right)\right] \gamma_{\mu} \\
& +\frac{F(k)}{2} \frac{k^{\mu}}{k^{2}}\left[\left[A\left(p_{2}+k\right)-A\left(p_{2}\right)\right]\left(2 \not p_{2}+\not k\right)-2\left[B\left(p_{2}+k\right)-B\left(p_{2}\right)\right]\right] .
\end{aligned}
$$

Notice that the above Ansatz satisfies the STI of Eq. (2.7) when $H=1$. Again, due to the transversality of $\Delta_{\mu v}(k)$, the second term on the rhs of Eq. (3.3), which is proportional to the longitudinal momentum $k^{\mu}$, does not contribute in the Eq. (3.1).

Then, inserting into Eq. (3.1) the propagators of Eq. (3.2) and the Ansatz for the quark-gluon vertex given by Eq. (3.3), it is straightforward to derive the following expression for $H$

$$
H^{[1]}\left(q, p_{2},-p_{1}\right)=1+i \frac{g^{2} C_{A}}{4} \int_{k} \mathscr{K}\left(p_{2}, q, k\right) f\left(p_{2}, q, k\right),
$$


where

$$
\mathscr{K}\left(p_{2}, q, k\right)=\frac{D(k-q) F(k) \Delta(k)\left[A\left(p_{2}+k\right)+A\left(p_{2}\right)\right]}{A^{2}\left(p_{2}+k\right)\left(p_{2}+k\right)^{2}-B^{2}\left(p_{2}+k\right)},
$$

while all spinorial structure is included in

$$
f\left(p_{2}, q, k\right)=A\left(p_{2}+k\right)\left[\not p_{2} q+\not k q-k \cdot q-\not p_{2} \not k \frac{q \cdot k}{k^{2}}\right]+B\left(p_{2}+k\right)\left[q-\not k \frac{q \cdot k}{k^{2}}\right] .
$$

\section{Quark symmetric configuration}

The projection of the form factors $X_{i}$, appearing in the definition (2.8), for arbitrary kinematics boils down to a complicated system of several equations. In order to make the problem at hand technically more tractable, we will only compute it in a specific kinematical limit: the quark symmetric limit where the quark momenta have the same magnitude and opposite signs, i.e. $p_{1}=-p / 2, p_{2}=p / 2$ and $q=-p$.

In this kinematical configuration, it is easy to see that only the (Dirac) form factor $L_{1}$ survives, and the vertex of Eq. (2.10) simplifies to

$$
\Gamma_{\mu}(-p, p / 2, p / 2)=L_{1} \gamma_{\mu} ; \quad \text { where } \quad L_{1} \equiv L_{1}\left(p^{2}, p^{2} / 4, p^{2} / 4\right) .
$$

In addition, the quark-ghost scattering kernel $H$ and $\bar{H}$, given by Eq. (2.8) and Eq. (2.9), simplify in this limit, and the terms proportional to $\not p$ become linearly dependent such that

$$
\begin{aligned}
H(-p, p / 2, p / 2) & =X_{0} \mathbb{I}+\left(X_{2}-X_{1}\right) \not p / 2, \\
\bar{H}(p,-p / 2,-p / 2) & =\bar{X}_{0} \mathbb{I}+\left(\bar{X}_{1}-\bar{X}_{2}\right) \not p / 2 .
\end{aligned}
$$

Setting $p_{1}=p_{2}=p / 2$ and $q=-p$ in Eq. (3.4), and taking the appropriate traces, it is straightforward to derive the following expressions for the form factor $X_{0}$ and the subtraction $X_{2}-X_{1}$ (in the Euclidean space)

$$
\begin{aligned}
X_{0}(p) & =1+\frac{g^{2} C_{A}}{8} \int_{k} \frac{D(p+k) F(k) \Delta(k) A_{2}\left[A_{2}+A_{1}\right]}{A_{2}^{2}(p / 2+k)^{2}+B_{2}^{2}}\left[p^{2}-\frac{(p \cdot k)^{2}}{k^{2}}\right], \\
X_{2}(p)-X_{1}(p) & =\frac{g^{2} C_{A}}{2 p^{2}} \int_{k} \frac{D(p+k) F(k) \Delta(k) B_{2}\left[A_{2}+A_{1}\right]}{A_{2}^{2}(p / 2+k)^{2}+B_{2}^{2}}\left[p^{2}-\frac{(p \cdot k)^{2}}{k^{2}}\right],
\end{aligned}
$$

where $A_{1}=A(p / 2), A_{2}=A(p / 2+k), B_{1}=B(p / 2)$, and $B_{2}=B(p / 2+k)$. Due to the fact that the momenta $p_{1}$ and $p_{2}$ have the same magnitude in this configuration, it is possible to show from the definition (2.9) that $X_{0}=\bar{X}_{0}$ and $X_{2}-X_{1}=\bar{X}_{2}-\bar{X}_{1}$.

Then, it is straightforward to see, from Eq. (2.12), that in the symmetric quark limit $L_{1}$ simplifies to

$$
L_{1}=F(p) X_{0}(p) A(p / 2)+F(p)\left[X_{2}(p)-X_{1}(p)\right] B(p / 2) .
$$

As we have seen in Eq. (4.3), both form factors $X_{0}$ and $X_{2}-X_{1}$ depend on the nonperturbative form of the four basic Green's functions, namely $\Delta(q), F(q), A(p)$ and $B(p)$. Therefore, in order 

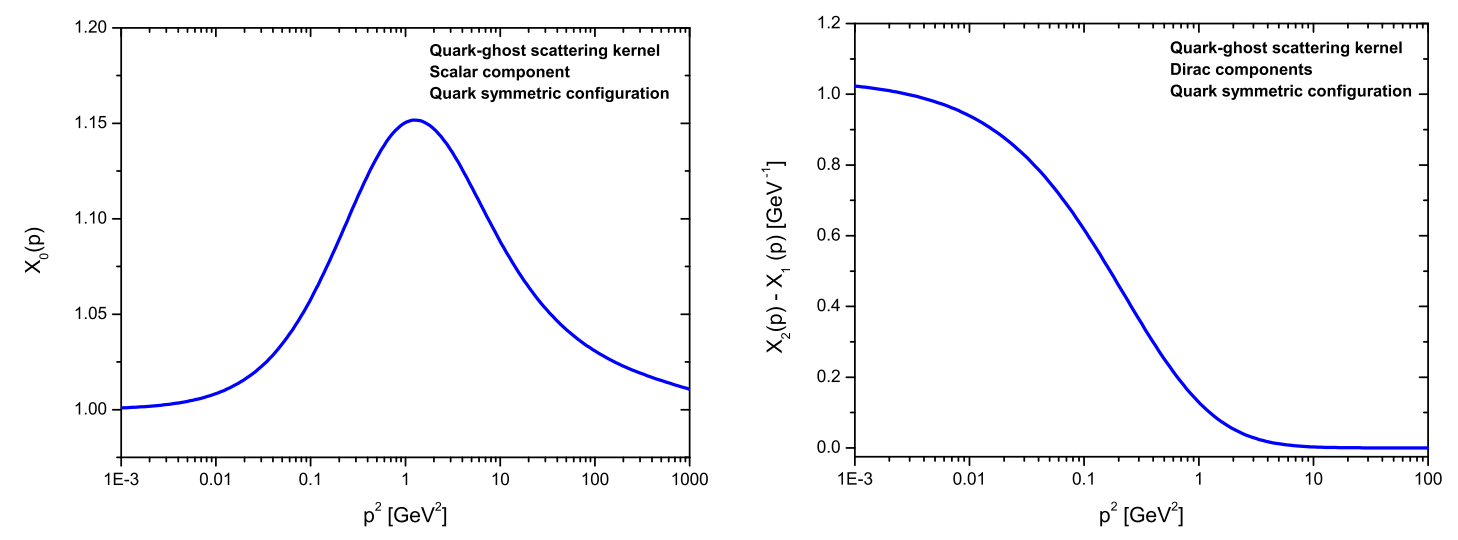

Figure 4: The scalar form factor $X_{0}$ (left panel) and the combination of the form factors $X_{2}-X_{1}$ (right panel) in the symmetric quark configuration for $\alpha\left(\mu^{2}\right)=g^{2} / 4 \pi=0.30$.

to proceed with the numerical analysis, we use for $\Delta(q)$ and $F(q)$ the lattice data obtained by [2], while for $A(p)$ and $B(p)$ we use the solution of the quark gap equation obtained in Ref [13]. All these functions were renormalized at $\mu=4.3 \mathrm{GeV}$, and in all our calculations we have fixed $\alpha\left(\mu^{2}\right)=g^{2} / 4 \pi=0.30$.

In Fig. 4 , we show the numerical results for $X_{0}(p)$ (left panel), and $X_{2}(p)-X_{1}(p)$ (right panel). On the left panel of Fig. 4, we can see that $X_{0}$ shows a maximum located in the intermediate momentum region (around $1-2 \mathrm{GeV}^{2}$ ), while in the UV and IR regions the curve goes to its perturbative value i.e. $X_{0} \rightarrow 1$.

On the right panel of Fig. 4 we notice that the combination $X_{2}-X_{1}$ saturates at a finite value in the deep IR region, while in the UV it vanishes asymptotically.

With all ingredients available, we are now in position to determine the behavior of the Dirac form factor $L_{1}$ in the symmetric quark configuration.

In Fig. 5 we show the result for $L_{1}$ obtained from Eq. (4.4). As we can clearly see, $L 1$ develops a sizable plateau in the IR region, and as expected, it recovers its perturbative value in the deep UV region.

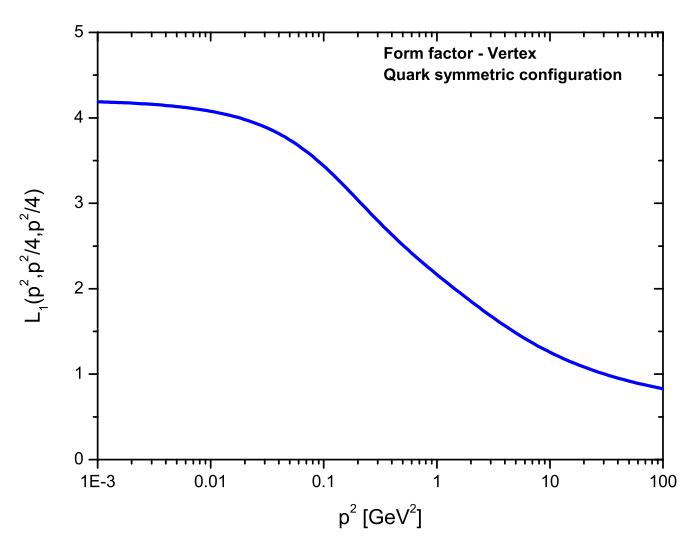

Figure 5: Numerical result for the vertex form factor $L_{1}$ in the quark symmetric configuration when $\alpha\left(\mu^{2}\right)=0.3$. 


\section{Conclusions}

We have presented the general methodology for determining the longitudinal form factors of the quark-gluon vertex from the fundamental STI that it satisfies. A key ingredient in this analysis is the quark-ghost scattering kernel, $H$ and its conjugate $\bar{H}$, whose field-theoretic origin and basic kinematic properties are rather subtle.

The first nonperturbative estimate of the form factors comprising $H$ has been computed using the "one-loop dressed" approximation of the corresponding integral equation, under certain reasonable dynamical assumptions. For the purposes of this presentation we have limited our analysis to the particular kinematic limit known as "quark symmetric configuration", which gives rise to considerable technical simplifications. The Dirac form factor of the quark-gluon vertex, $L_{1}$, has been obtained in this particular kinematic configuration, and its basic features have been studied. The methodology presented here may be directly extended to arbitrary kinematic configurations, furnishing valuable information on such a fundamental quantity as the quark-gluon vertex.

\section{Acknowledgments}

We would like to thank the organizers of the Xth Quark Confinement and the Hadron Spectrum for the pleasant conference. The research of ACA is supported by CNPq under the grants 305850/2009-1, 453118/2010-0, and by FAPESP - grant 2012/15643-1. The work of J. P. is supported by the Spanish MEYC under grant FPA2011-23596.

\section{References}

[1] A. Cucchieri and T. Mendes, PoS LAT2007, 297 (2007); Phys. Rev. Lett. 100, 241601 (2008).

[2] I. L. Bogolubsky, E. M. Ilgenfritz, M. Muller-Preussker and A. Sternbeck, PoS LAT2007, 290 (2007).

[3] O. Oliveira and P. J. Silva, PoS LAT2009, 226 (2009).

[4] A. C. Aguilar, D. Binosi and J. Papavassiliou, Phys. Rev. D 78, 025010 (2008).

[5] D. Binosi and J. Papavassiliou, Phys. Rept. 479, 1 (2009).

[6] C. S. Fischer, J. Phys. G 32, R253 (2006).

[7] D. Dudal, J. A. Gracey, S. P. Sorella, N. Vandersickel and H. Verschelde, Phys. Rev. D 78, 065047 (2008).

[8] C. D. Roberts and A. G. Williams, Prog. Part. Nucl. Phys. 33, 477 (1994).

[9] A. Bashir, R. Bermudez, L. Chang and C. D. Roberts, Phys. Rev. C 85, 045205 (2012).

[10] M. S. Bhagwat and P. C. Tandy, Phys. Rev. D 70, 094039 (2004) .

[11] A. Kizilersu, D. B. Leinweber, J. -I. Skullerud and A. G. Williams, Nucl. Phys. Proc. Suppl. 141, 244 (2005); Eur. Phys. J. C 50, 871 (2007).

[12] J. -I. Skullerud, P. O. Bowman, A. Kizilersu, D. B. Leinweber and A. G. Williams, Nucl. Phys. Proc. Suppl. 141, 244 (2005).

[13] A. C. Aguilar and J. Papavassiliou, Phys. Rev. D 83, 014013 (2011).

[14] A. I. Davydychev, P. Osland and L. Saks, Phys. Rev. D 63, 014022 (2001).

[15] J. S. Ball and T. W. Chiu, Phys. Rev. D 22, 2542 (1980). 